\title{
AUTOMORPHISM GROUPS EMITTING LOCAL ENDOMORPHISM NEAR-RINGS
}

\author{
GARY L. PETERSON
}

(Communicated by Donald Passman)

\begin{abstract}
The question of whether a group of automorphisms $A$ containing the inner automorphisms of a nonabelian $p$-group $G$ can generate a local endomorphism near-ring when $A$ is not a $p$-group is considered. Conditions on $A$ are obtained which give us that the endomorphism near-ring generated by $A$ is not local. An example is given showing that the endomorphism near-ring generated by $A$ can be local when these conditions are not met.
\end{abstract}

\section{INTRODUCTION}

Let $G$ be an additive (but not necessarily abelian) group and $S$ be a semigroup of endomorphisms of $G$. Under pointwise addition and composition, the set $R$ of all functions from $G$ to $G$ of the form $\varepsilon_{1} s_{1}+\cdots+\varepsilon_{n} s_{n}$, where $\varepsilon_{i}= \pm 1$ and $s_{i} \in S$ along with the zero map forms a distributively generated left near-ring when functions are written on the right called the endomorphism near-ring generated by $S$. If, in addition, $S$ contains the group of inner automorphisms $\operatorname{Inn}(G)$ of $G$, the endomorphism near-ring is said to be tame. The reader desiring a reference on near-rings which contains a treatment of the fundamental aspects of endomorphism near-rings may consult Meldrum's book [6].

A left near-ring $R$ is said to be local if

$$
L=\{r \in R \mid r \text { does not have a right inverse }\}
$$

is a right $R$-subgroup of $R$. Localness for tame endomorphism near-rings $R$ of finite groups $G$ was studied in [5]. Among the results obtained there were that if $R$ is local, then $G$ is a $p$-group. Furthermore it was shown that if $A$ is a $p$-group of automorphisms of a $p$-group $G$ containing $\operatorname{Inn}(G)$, then the endomorphism near-ring $R$ generated by $A$ is local and the question was raised as to whether the converse of this latter result holds for nonabelian $p$-groups. The purpose of this paper is to study the converse.

Received by the editors June 1, 1988. Presented at the 95th Annual Meeting of the American Mathematical Society, January 11, 1989.

1980 Mathematics Subject Classification (1985 Revision). Primary 16A76; Secondary 20D45, 20E36, $20 \mathrm{~F} 28$.

Key words and phrases. Endomorphism near-ring, local near-ring. 
To describe our results, let $A$ be a group of automorphisms of a nonabelian $p$-group $G$ containing $\operatorname{Inn}(G)$ and let $R$ be the endomorphism nearring generated by $A$. If $R$ is local, we have from Theorem 2.3 of [5] that $\operatorname{Aut}(G) \cap R(\operatorname{Aut}(G)$ the automorphism group of $G$ ) has a normal $p$-Sylow subgroup which has a cyclic complement. It then follows that $A$ must possess the same properties. Consequently, we may restrict our attention to such automorphism groups; that is, we may assume that $A$ has the form

$$
A=K P \text {, }
$$

where $P$ is a $p$-Sylow subgroup of $A$ with $P \triangleleft A, K$ is cyclic, and $K \cap P=1$. Let $K=\langle\alpha\rangle$ and $|K|=k$. If $H \subseteq L$ are $R$-submodules of $G$ with $L / H$ a minimal $R$-module, we shall say that $L / H$ is an $R$-principal factor of $G$. Suppose that $p^{n}$ is the order of some $R$-principal factor of $G$. We will show

Theorem. If $k$ does not divide $p^{i}+p^{j}-1$ for each $0 \leq i, j \leq n-1$, then $R$ is not local.

Section 2 will be devoted to the proof of this result and in $\S 3$ we will give an example to show that $R$ can be local if $k$ does not satisfy the hypothesis of the theorem. The method that we shall use in our proof is similar to one of the techniques employed by G. Higman in his characterization of Suzuki 2-groups (cf. [2, Lemma 4]) and the example in $\S 3$ is itself a Suzuki 2-group.

\section{Proof of the theorem}

Suppose that the theorem is false. Let $G$ be a nonabelian $p$-group and $A$ be a group of automorphisms of $G$ of the prescribed form for which the endomorphism near-ring $R$ generated by $A$ is local. Let $G=\gamma_{1}(G)>\gamma_{2}(G)>$ $\cdots$ be the lower central series of $G$. Set $L_{1}=G /\left(p G+\gamma_{2}(G)\right)$ and $L_{2}=$ $\gamma_{2}(G) /\left(p \gamma_{2}(G)+\gamma_{3}(G)\right)$ which are then vector spaces over $\mathbf{Z}_{p}$. Let

$$
L_{10}=0<L_{11}<L_{12}<\cdots<L_{1 m}=L_{1}
$$

and

$$
L_{20}=0<L_{21}<L_{22}<\cdots<L_{2 l}=L_{2}
$$

be $R$-principal series of $L_{1}$ and $L_{2}$, respectively. By Theorem 2.2 of [5], we have that each factor $L_{i j+1} / L_{i j}$ is $R$-isomorphic to $R / J_{2}(R)$ and $R / J_{2}(R)=$ $G F\left(p^{n}\right)$. Moreover, it follows from Theorem 2.3 of [5] that $P$ acts trivially and $K$ acts faithfully on each factor $L_{i j+1} / L_{i j}$. The trivial action of $P$ on $L_{i j+1} / L_{i j}$ gives us that $L_{i j+1} / L_{i j}$ is an irreducible $K$-module. Since $K$ acts faithfully on $L_{i j+1} / L_{i j}$, any eigenvalue of $\alpha$ on $L_{i j+1} / L_{i j}$ is a primitive $k$ th root of unity ([1], Lemma 5.6.3).

Now consider the action of $K$ on $L_{1}$. By Maschke's Theorem, we can write

$$
L_{1}=H_{1} \oplus H_{2} \oplus \cdots \oplus H_{m}
$$

where $H_{1}=L_{11}$ and, for $2 \leq r \leq m, H_{r}$ is isomorphic to $L_{1 r} / L_{1 r-1}$ as a $K$ module. Let $\lambda$ be a primitive $k$ th root of unity and $F=\mathbf{Z}_{p}[\lambda]=G F\left(p^{n}\right)$. The 
eigenvalues of $\alpha$ on $H_{r}, 1 \leq r \leq m$, are the conjugates $\lambda_{i}=\lambda^{p^{i}}$, where $i=$ $0,1, \ldots, n-1$. Set $L_{i}^{\prime}=L_{i} \otimes F, i=1,2$, where the tensor products are over $\mathbf{Z}_{p}$. For each $1 \leq r \leq m$, we can find a basis of eigenvectors $u_{0 r}, \ldots, u_{n-1 r}$ of $H_{r} \otimes F$ relative to $\alpha \otimes 1$ with $u_{i r}(\alpha \otimes 1)=\lambda_{i} u_{i r}$.

Commutators give rise to a $\mathbf{Z}_{p}$-bilinear map from $L_{1} \times L_{1}$ onto $L_{2}$ by letting

$$
\left(g_{1}+p G+\gamma_{2}(G), g_{2}+p G+\gamma_{2}(G)\right) \rightarrow\left[g_{1}, g_{2}\right]+p \gamma_{2}(G)+\gamma_{3}(G)
$$

(cf. [3, VIII, 6.1]). This map extends to a $F$-bilinear map from $L_{1}^{\prime} \times L_{1}^{\prime}$ onto $L_{2}^{\prime}$ when we set

$$
\begin{gathered}
{\left[\left(g_{1}+p G+\gamma_{2}(G)\right) \otimes f_{1},\left(g_{2}+p G+\gamma_{2}(G)\right) \otimes f_{2}\right]} \\
=\left(\left[g_{1}, g_{2}\right]+p \gamma_{2}(G)+\gamma_{3}(G)\right) \otimes f_{1} f_{2},
\end{gathered}
$$

where $f_{1}$ and $f_{2} \in F$. Since the eigenvectors $u_{i r}, 0 \leq i \leq n-1,1 \leq r \leq m$ form a basis for $L_{1}^{\prime}$, it follows that the vectors $\left[u_{i r}, u_{j s}\right]$ span $L_{2}^{\prime}$. As

$$
\left[u_{i r}, u_{j s}\right](\alpha \otimes 1)=\lambda^{p^{i}+p^{j}}\left[u_{i r}, u_{j s}\right]
$$

the eigenvalues of $\alpha$ on $L_{2}$ are found among $\lambda^{p^{i}+p^{j}}, 0 \leq i, j \leq n-1$.

Since $L_{11}$ and $L_{21}$ are $R$-isomorphic, we have that $\lambda$ is an eigenvalue of $\alpha$ on $L_{21}$. But then $\lambda=\lambda^{p^{i}+p^{j}}$ for some $0 \leq i, j \leq n-1$ which implies $k$ divides $p^{i}+p^{j}-1$ for some such $i$ and $j$ contrary to our assumption.

\section{AN EXAMPLE}

We now give an example showing that $R$ can be local if the condition on $k$ in our theorem does not hold. Let $F=G F\left(2^{4}\right)$ and let $\lambda$ be a generator for the multiplicative group of $F$. The order of $\lambda$ is then 15 . Set $\beta=\lambda^{3}$ which has order 5. As the conjugates of $\beta$ are $\beta, \beta^{2}, \beta^{4}$, and $\beta^{8}=\beta^{3}$ and as $\left[F: \mathbf{Z}_{2}\right]=4$, we have $F=\mathbf{Z}_{2}[\beta]=\mathbf{Z}_{2}\left[\beta^{3}\right]$.

Let

$$
G=\left\{\left[\begin{array}{ccc}
1 & 0 & 0 \\
a & 1 & 0 \\
b & a^{2} & 1
\end{array}\right] \mid a, \quad b \in F\right\} .
$$

For convenience, set

$$
u(a, b)=\left[\begin{array}{ccc}
1 & 0 & 0 \\
a & 1 & 0 \\
b & a^{2} & 1
\end{array}\right] .
$$

$G$ is a group under matrix multiplication which in additive notation becomes

$$
u(a, b)+u\left(a^{\prime}, b^{\prime}\right)=u\left(a+a^{\prime}, b+b^{\prime}+a^{\prime} a^{2}\right) .
$$

Define $\alpha$ on $G$ by

$$
u(a, b) \alpha=\left(\beta a, \beta^{3} b\right)
$$

which is easily checked to be an automorphism of $G$. Let $A=\langle\alpha\rangle \operatorname{Inn}(G)$ and $R$ be the endomorphism near-ring generated by $A$. Let $N=\{u(0, b) \mid b \in F\}$. 
Then $N$ and $G / N$ are $R$-modules. Moreover, they are irreducible $R$-modules since $F=\mathbf{Z}_{2}[\beta]=\mathbf{Z}_{2}\left[\beta^{3}\right]$. Thus $0<N<G$ is an $R$-principal series of $G$. Note that $|N|=|G / N|=2^{4}$ and $|\alpha|=5=2+2^{2}-1$ so that the condition of the theorem is indeed violated.

We now argue that $R$ is local. Since $0<N<G$ is the socle series of $G$, it suffices to show that $N \simeq G / N \simeq R / J_{2}(R)$ as $R$-modules by Theorem 2.3 of [7]. In fact, we need only show $N \simeq G / N$. For once we have this isomorphism, we will have $\operatorname{Ann}_{R}(N)=\operatorname{Ann}_{R}(G / N)$. Because $J_{2}(R)$ is the annihilator of the socle series of $G\left[4\right.$, Lemma 2.5], we then will have $J_{2}(R)=\operatorname{Ann}_{R}(G / N)$, whence $G / N \simeq R / J_{2}(R)$.

To get our isomorphism, define $\varphi: G / N \rightarrow N$ by

$$
(u(a, b)+N) \varphi=u\left(0, a^{8}\right) .
$$

It is easily checked that $\varphi$ is a group isomorphism. To see that $\varphi$ is an $R$ isomorphism it suffices to show that $\alpha$ commutes with $\varphi$ since $\operatorname{Inn}(G)$ acts trivially on both $G / N$ and $N$. But this follows since

$$
\begin{aligned}
(u(a, b)+N) \alpha \varphi & =\left(u\left(\beta a, \beta^{3} b\right)+N\right) \varphi=u\left(0, \beta^{8} a^{8}\right)=u\left(0, \beta^{3} a^{8}\right) \\
& =(u(a, b)+N) \varphi \alpha
\end{aligned}
$$

and our argument is complete.

\section{REFERENCES}

1. D. Gorenstein, Finite groups, Harper and Row, New York, 1968.

2. G. Higman, Suzuki 2-groups, Illinois J. Math. 7 (1963), 79-96.

3. B. Huppert and N. Blackburn, Finite groups. II, Springer-Verlag, Berlin. 1982.

4. C. Lyons and J. Meldrum, Characterizing series for faithful d. g. near-rings, Proc. Amer. Math. Soc. 72 (1978), 221-227.

5. C. Lyons and G. Peterson, Local endomorphism near-rings, Proc. Edinburgh Math. Soc. 31 (1988), 409-414.

6. J. Meldrum, Near-rings and their links with groups, Pitman, London, 1985.

7. G. Peterson, On the structure of an endomorphism near-ring, Proc. Edinburgh Math. Soc. (to appear).

Department of Mathematics and Computer Science, James Madison University, HARRISONBURG, VIRGINIA 22807 